\title{
Optics demonstrations with an overhead projector
}

\section{Douglas Goodman}

Douglas S. Goodman, "Optics demonstrations with an overhead projector," Proc. SPIE 2525, 1995 International Conference on Education in Optics, (13 October 1995); doi: 10.1117/12.224000

SPIE Event: SPIE's 1995 International Symposium on Optical Science, SPIE. Engineering, and Instrumentation, 1995, San Diego, CA, United States 


\title{
Optics Demonstrations with an Overhead Projector
}

\author{
Douglas S. Goodman \\ Polaroid \\ Optical Engineering Department \\ 38 Henry St. \\ Cambridge, MA 02139-4894
}

Magic Lantern, John Zahn, 1685

From Eugene Ostroff, Smithsonian

"Photographic Enlarging: A History,"

Photographica Journal, Vol 1, No 3 (1984)

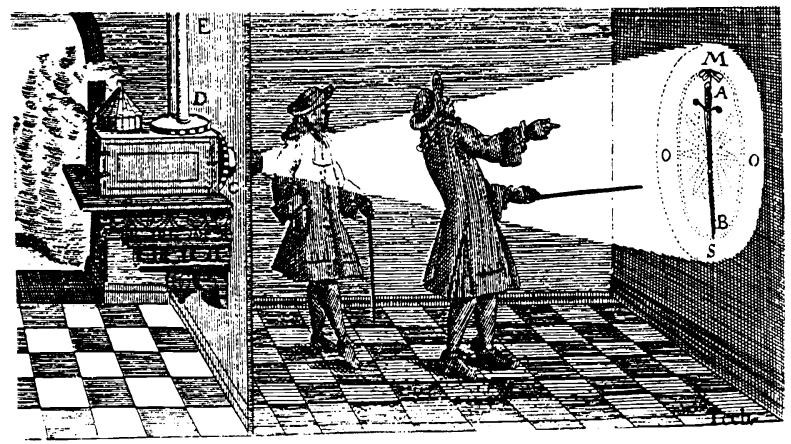

The idea for these demonstrations occurred when, while preparing a demonstration for a course, I realized that I was reinventing the overhead projector. Because the projector is so readily available, I undertook to see how much could be done with it using additional inexpensive and readily available materials. No fundamental novelty is claimed, since projectors have been around for at least 300 years, and people have probably tried most everything. Moreover, most of these demonstrations are variations on classics that can be done in many ways. The purposes of this work are to point out just how much can be done so easily on the projector, to provide guidance in optimizing the demonstrations, and to provide information on vendors and useful materials. Most important, I hope to encourage people to do demonstrations. The projector is obviously ideal for many demonstrations of human vision, but I have not undertaken these, leaving them to specialists in the field.

\section{INTRODUCTION}

Demonstrations are good, overhead projectors are common and inexpensive, and much can be done with the projector quickly, easily, and with inexpensive materials.

\section{The Importance of Demonstrations}

People are visual creatures. Much of the brain is devoted to vision. People are attracted to bright lights, autumn leaves, television, computer graphics, and visual stimuli of all sorts. Language is filled with visual metaphors, an example being the term "color" as used in music. Appearances are important. Seeing is believing, remembering, and reinforcing. Now, in the Age of Simulation, demonstrations have new importance as a counterbalance to complete dependence on and faith in what computers-with their alluring visual outputs-produce.

\section{Advantages of the Overhead Projector}

Overhead projectors are commonplace, and inexpensive, with prices starting at about $\$ 250$. Expensive projectors are not necessary for demonstrations, and, indeed, projectors with "bad" performance can be used to show aberrations. Projectors are safe- UL approved and built for general use. Projectors supply a major portion of many experimental setups. In particular, they contain a strong lamp, with cooling, electrical power supply, and condensing lens a substantial part of the apparatus that for many optical demonstrations is the most trouble and least interesting.

The additional materials required for most of the demonstrations described here are inexpensive, some being everyday objects. Many of the purchased items are obtained from educationa—as opposed to more pricy scientific - vendors, as well as, for example, theatrical supply stores. Many of the fabricated parts are plastic that can be made in a machine shop or carpentry shop, buffed smooth, rather than figured from glass in an optical shop. 
Demonstrations are not, in themselves, associated with any educational level or age group. This is a matter of how they are discussed and explained. Demonstrations can be presented in a number of ways. Youngest audiences can be approached with a 'gee whiz' treatment, emphasizing that the world is interesting and surprising. Demonstrations can be used to cultivate curiosity, with the participants playing to see what happens. Demonstrations can be used as reinforcements to lecture material. Some demonstrations are directly related to applications, for instance, metrology. Here instrumentation, rather than 'pure' physics is concentrated upon, providing appeal for 'practically minded' students. Some demonstrations are related to appearances, why things look as they do, curiosity about which is to be nurtured, since the environment provides endless objects of study. A number of concepts that might be considered advanced can be introduced with demonstrations, possibly without explicit mention for junior audiences. These include symmetry, special orientations, parity, resolution, convolution, and differential measurement.

These demonstrations are designed to be done quickly, so they can be inserted in a lecture in 'real time.'

Whenever possible, the demonstrations employ comparisons, for instance, between a beam of light that is acted on somehow and a "control" beam. Motion and change are used to capture attention, and colors for aesthetic appeal.

These demonstrations can be done in ways other than the standard "teacher in the front" mode. They can be done as student experiments or presented to the students as problems or even oral exam questions.

\section{The Projector and the Laser}

When the laser appeared, some demonstrations and experiments, especially those of interference and diffraction, became very much simpler, so they began to be done with lasers. The laser became more familiar and less expensive and began to be used increasingly, while important non-laser demonstrations were forgotten. The overhead projector demonstrations complement those of the laser very nicely; what the laser does best cannot be done on the projector, and the projector does much that the laser cannot do at all, an obvious example being white light demonstrations.

\section{The Overhead Projector}

Overhead projectors differ enough to affect the optimization of some of these demonstrations. Consequently, you should familiarize yourself with a projector before undertaking a demonstration. Most of these demonstrations either cannot be done well or cannot be done at all with the portable reflected light overhead projectors that have a Fresnel mirror on their platen..

A study of the projector is worthwhile since the principles and components of projectors are common to many instruments. Lift the hood and see what is inside. Different projectors can be compared to see the various ways in which the same problem has been treated, as well as methods of inexpensive mass production. In addition to the optics, there is electrical power, cooling, safety interlocks, mechanical mechanisms for lamp switching and lamp focus. The optics systems include the lamp, illumination optics, imaging optics, and a mirror.

The illumination optics captures as much of the lamp's output as possible, produces reasonably uniform irradiance at the platen, and directs the light into the imaging lens. The Fresnel lens under the platen images the lamp in to the lens. Show the lamp by holding an additional lens projector and the screen, where the lamp is imaged. Improve the quality of the lamp image by covering the platen with a piece of cardboard with a hole cut out, to stop down the Fresnel lens, thereby reducing its aberrations.

The mirror performs three functions. (1) It produces a vertical image of a horizontal transparency. (2) It permits the speaker to face the audience while the transparency is seen upright. (Bowling alley projectors, for which everyone faces the same direction, are different.) (3) It reverses parity. The speaker and audience see the transparency and its image in opposite directions relative to the projector axis. 
To demonstrate the parity change produced by a mirror, hold a mirror just after the projector lens, producing an image on the wall, floor, or ceiling of reversed parity. Move the mirror to show that the image parity stays the same, although its orientation changes.

A part of the projection system is the screen, which is designed to scatter the light incident upon it into a range of angles subtended by the audience. Show the screen's angular scattering properties by taping different materials to it, for example white paper and aluminum foil. Walk around the room to see how the relative brightness of the different surfaces change with position.

\section{Some Practical Tips}

- Block out the irrelevant portion of the illuminated field with a platen field stop to eliminate space in which nothing is happening, to concentrate attention, and to reduce the light in the room. A generally useful stop is a white cardboard sheet covering the platen with a rectangular hole about 7" $\times 9 "$. Dark cardboard absorbs and warms.

- Energy densities are greatest where the lamp is focused, usually just below the projection lens, so be careful about placing color filters, polarizers, and other materials there. Plastic can melt and paper can ignite. This can be used to show that radiation transfers energy, for example, by putting a beaker of water with a thermometer at the focus. To reduce the power at the focus, block some of the light with a field stop on the platen or place on the platen an $\mathbb{R}$ absorber, for instance one of the sheets used with theatrical lights.

- Eliminate distracting stray light emitted from various openings in the projector base. This light is not noticed in ordinary use, but is detrimental to demonstrations. To check for stray light, cover the platen completely and turn on the projector in a dark room. In obstructing these openings, do not block cooling air. A loop of tape often does the job.

- Many demonstrations require additional field stops that can be cut from thin cardboard. A particularly useful one has a single hole in the center about $1 \mathrm{~cm}$ in diameter. Another has a slit the same width. A cutout letter $\mathbf{F}$, or something else that shows parity, is useful.

- Eliminate unwanted moiré effects involving the projector Fresnel lens by raising the demonstration material on a sheet of glass or plastic and refocusing.

- Color filters sold by theatrical supply houses are most generally useful. They are large, relatively inexpensive, and are designed to withstand heat. Rosco sells a "swatch book" of filter samples with spectral transmission curves.

- "Ripply glass," such as that used in shower doors is useful. It can be obtained from stores that supply hobbyists who work in stained glass windows, Tiffany-type lamps, and so forth. The appearance on the projector of these materials is difficult to predict, so it is best to get a variety. (This material can produce fascinating diffraction patterns when illuminated by a laser.)

- A strong diffuser on the platen results in too weak an image, since too much light is scattered beyond the lens. A useful diffuser is Rosco \#110.

- Projectors can be used backwards, by using the lens to image onto the platen, covered with a suitable screen material, for instance, white paper.

- For many experiments requiring two projectors, one can be a slide projector. 
- Pairs of projectors can be used in a number of ways. Two patterns, images, or colors can be overlaid. One projector can be used to produce an image on a screen, and another to reimage it onto the platen. For some demonstrations, the second projector can be a slide projector.

- An object that must be vertical can be projected as follows. Place it in the center of the platen. On one side, set a mirror at $45^{\circ}$ to produce an image in the platen plane. On the other side set up a mirror angled to direct light from the base through the object and into the projection lens.

- Darkfield illumination can be obtained and objects can be illuminated transversely from one or more directions by using mirrors on the platen and an opaque sheet under the object. However, this is inefficient.

- The projector base can provide illumination for other demonstrations. If the lens is removed, the application can be placed above the base. Alternately, a mirror can be used to redirect the light horizontally.

- The projector base can produce a somewhat collimated beam. Find the location on the platen of the best beam empirically by blocking most of the platen with a stop that is moved about it.

- A light table can be made by covering the base of the projector with a sheet of milky plastic or opal glass.

- Many transparencies can be made easily, without photographic processing. Transparencies can be made on thermal copiers and on many photocopiers, so patterns found in books and catalogues, for example resolution targets and patterns that produce good moire effects, can be used. Copiers with enlarging and reduction capabilities are available. Computer graphics can be readily produced..

- A turntable with a hole in the center can be made from Rubbermaid ${ }^{\mathrm{TM}}$ lazy susans sold in supermarkets for a few dollars. Holes, which need not be precise, can be cut in the centers.

\section{MONOCHROMATIC GEOMETRIC OPTICS}

\section{Plane Parallel Plate}

To show axial image shift, place a grid on half the platen and above it a glass or plastic plate or a beaker of water. Refocus the lens to show an axial shift. Hold another grid next to the plate, and move it axially until it is in focus, showing the location of the image and that the axial shifting does not change lateral scale.

Show translation by tilting a clear plate over a marked transparency. Use different thickness plates, side by side if possible, to show that translation varies with thickness for a given angle. An application is the optical micrometer, in which small image shifts are measured by tilting a thin plate over an angle large enough to be easily measured.

Demonstrate a scanner using a plastic cube with two handles on opposite sides. Cover the platen except for a single hole, and the rotate the cube with the handles. An application is in high-speed film cameras where the film does not come to rest to expose each frame.

\section{Thin Prism}

Show the monochromatic properties of the thin prism with a clear plastic prism, about $100 \mathrm{~mm} \times 100 \mathrm{~mm}$ with an apex angle of about $10^{\circ}$. On the platen place a cardboard with a letter " $\mathrm{F}$ " cut out, and cover the opening with a green filter, since dispersion is not to be shown here. Hold the prism at the projector lens. Tilt the prism to change the deviation angle and to show the minimum deviation angle. Rotate the prism 
to move the image on an arc without rotating it, as a mirror would do. Compare prisms of the same material but different apex angles and prisms with the same apex angle made of different materials. Move a prism across the projector lens pupil to produce two displaced images with varying amounts of light. A variable-angle prism can be made by placing together two thin prisms and rotating them.

\section{Patterns in Shallow Water}

Simulate caustic patterns like those seen at the bottom of shallow water by placing on the platen a piece of ripply glass of the kind used in shower doors. Tilt the glass to show the change of contrast with depth of water.

\section{Total Internal Reflection}

Show total internal reflection with a plastic $45^{\circ}$ prism, placed over a hole in a piece of cardboard on the platen. A piece of diffusing tape on the output face serves as a screen to make the outgoing beam visible. Cut one end of a plastic bar at about $45^{\circ}$ and smooth the end. Hold the angled end near the lamp image and light traverses the bar laterally. Internal reflection is shown with fluorescent plastic sheets, as described below.

\section{Retroreflector}

With an open platen, hold a plastic bicycle reflector at the screen and its observe its appearance from different places in the room. Similarly, hold retroreflective material against the screen. The screen itself, as discussed above, is somewhat retroreflective.

\section{DISPERSION}

Show dispersion with a glass prism at the projection lens, with the platen covered except for a small central hole. A chromatically dispersed image of the hole is seen, and tilting the prism shows that the minimum deviation angle varies with wavelength. Show that refraction angles vary with material by using a pair of prisms of different materials with the same angle. A plastic prism can be made to match the shape of an existing glass one. Cover the platen with a cardboard from which a slot has been cut. The resulting spectrum on the screen is curved, since the rays from the various points in the slot make different angles with the prism. Slits in spectrometers are curved for this reason. Another object with which to show dispersion is a plastic cone with a large apex angle, which gives a colored circle on the screen.

The chromatic aberration of the projector Fresnel lens provides another demonstration. Block the platen except for an opening near an edge, and hold a white card under the projector lens to see a chromatically aberrated image of the lamp.

\section{Rainbow}

The rainbow can be demonstrated with a single plastic ball. Hold a viewing screen near the projection lens on the audience side, and hold the ball in the light leaving the projector to produce a rainbow-like pattern on the screen. This requires some experimentation. There is not much light, so reduce stray light with a platen cardboard from which part of an annulus is cut out, blocking much of the light that does not contribute to the rainbow. Use a light blue color correction filter on the platen to enhance the blue end of the spectrum. An analogous demonstration can be done with a plastic cylinder and a field stop with a straight slot.

\section{IMAGING}

Record images by projecting onto paper taped to the wall or onto a whiteboard or a blackboard. Images can be traced and measurements made with rulers and protractors. 


\section{Miscellaneous Properties}

Measure transverse magnification. by putting a clear plastic ruler on the platen, measuring its image, and finding the ratio.

Show orthographic imaging and keystone distortion with a clear plastic grid on the platen projected onto a screen at various angles. Tilt the object grid over the platen, and measure the sizes and angles of image features.

Rotate the transparency and show that the image rotates identically.

Demonstrate front and rear projection and parity by projecting an appropriate pattern on a screen that can be viewed from both sides, for instance tracing paper or thin cloth. Flip over the transparency on the platen to show parity reversal, and rotate the transparency in the platen plane to distinguish between orientation and parity.

Show vignetting with a piece of cardboard with a hole cut out, held at various places between the platen and lens.

To show imaging reversibility, turn the projector off, cover the platen with a white paper to act as a screen, and image a bright external object onto the platen.

Introduce auxiliary lenses either before or after the projector lens to show how image size and location change. Translate and tilt these lenses to show other properties.

To show relationships between the distances between object and lens and between lens and image, move the transparency above the platen, move the projection lens, and move the projector as a whole toward or away from the screen.

\section{Resolution and Empty Magnification}

To demonstrate resolution limitations, use a transparency with a variety of feature sizes. One can be made by photocopying a picture of a resolution target from a book or catalogue. View the image from near the screen to observe that images of smaller features become blurrier and finally indistinguishable. With a good projection lens, the resolution of an image viewed from the normal audience position is limited by that of the viewer's eye, so the limitations of the projection lens are not observable. Project a piece of microfiche so that its geometrical image is sufficiently large that the letters ought to be readable. They remain unreadable, even when observed at the screen with a magnifier. Magnification and resolution are not matched.

\section{Phase Structures}

Various properties of phase structure images can be shown with ripply glass. If a grid on the platen is covered with a weak ripply glass, the image is distorted, but remains sharp. If the glass is moved to the lens, the image is blurry. This shows the difference between looking upward through the atmosphere from the earth's surface and looking downward from above, as from a satellite. Move the ripply glass between the platen and the lens to show the transformation between the two extremes.

\section{Twinkling Stars - Imaging Through Turbulent Material}

Simulate turbulent media by translating ripply glass. To imitate the twinkling of stars, use weak "Aquatex" style ripply glass and a cardboard on the platen with several $5 \mathrm{~mm}$ holes to represent the stars.

\section{Phase Objects and Magnitude Objects}

Some objects, known as magnitude objects, such as a cardboard with holes, either pass or block light. Others, known as phase objects, such as ripply glass, pass all light, but deflect it in various directions. 
The images of magnitude and phase objects have different properties, especially as regards their dependence on illumination and changes through focus. Such properties, which are often seen most clearly with microscopes, can be demonstrated with the overhead projector. When a cardboard with holes is defocused, its image basically becomes a blurred version of what is seen in focus, but the image of a ripply glass changes in form. For a weakly rippled glass, image contrast may be greater out of focus, unlike a magnitude object. The defocused images of a magnitude object are nominally the same on both sides of focus, but this is not the case for phase objects. An object that combines both magnitude and phase attributes can be created by placing a ripply glass on a patterned cardboard.

\section{Defocus}

Defocus effects differ for projectors with different types of illuminations. Periodic objects when imaged defocused are nearly periodic, and make interesting patterns. Periodic plastic materials are fairly common and these can be tried. Tilt the materials on the platen to simultaneously show different amounts of defocus. Change the effects of defocus by adding a weak diffuser at the platen.

\section{Ray Aberrations}

Show that a blurred image can be decomposed into a number of differently shifted images. Hold a piece of cardboard with a hole about $5 \mathrm{~mm}$ in diameter at the projector lens and translate it. The image on the screen is sharp for each hole position, but it moves when the hole is moved. Thus rays passing through different parts of the lens aperture do not intersect the screen in the same place.

\section{Aberrations and Resolution}

Overhead projector aberrations can be increased with a diffuser on the platen that sends light through the full aperture of the lens, rather than through only the portion for which it is best corrected. Observe the image of a resolution target with and without a diffuser.

\section{Astigmatism}

Some projectors have enough astigmatism for a simple demonstration. Using a pattern with orthogonal sets of lines at the edge of the field, move the projection lens through focus to show that lines at different orientations are sharpest at different focal positions. With better corrected projectors, obtain astigmatism by placing above the pattern on the platen a cylindrical lens with a focal length of about $300 \mathrm{~mm}$. Weaken a stronger cylindrical lens by immersing it in a petri dish of water or oil. Orient the cylinder at an angle above a square pattern to make a parallelogram image.

\section{Chromatic Aberration}

Observe lateral color with a field stop that has a slit at the edge of the field parallel to the side of the platen. Stand near the screen to best see the colored image. Produce displaced images by covering half of the slit with a red filter and half with a blue one.

Observe chromatic aberration indirectly by using a color filter with a resolution test target, showing that the image is sharper when the wavelength range is decreased.

Observe the chromatic aberration of the Fresnel lens as described in the dispersion section above.

\section{Dividing the Pupil for Multiple Images and Special Effects}

Insert a thin prism part way across the projector lens pupil to produce double images. With a multi-part platen, one image can be made to change to another. Image changing can also be done with an object made of polarizers at different angles and a rotating analyzer at the lens. Likewise, an object can be composed of different color filters with a changing filter at the lens. 


\section{Knife-Edge Test}

The knife edge test can be demonstrated on a projector whose lamp has a small filament and no integral reflector. Raise the projector lens to make the filament image accessible, and introduce a heat-insensitive knife edge. The object is a piece of ripply glass, raised from the platen so as to produce a focused image with the raised lens.

\section{Projection Contour Determination}

Project a grid on a three dimensional diffuse object, e.g. a plaster of Paris shape, to show a method of determining contours.

\section{Contrast Reduction and Background Light}

Use one projector to produce an image on the screen, and another to provide a uniform background, showing the effects of contrast reduction. Relative irradiances can be varied. With color, there are additional possibilities.

\section{Face-to-Face Projectors}

With two projectors pointing at each other, several methods of re-imaging can be shown. With nothing between them, a small field of view can be obtained if the alignment between the two projectors is precise. A screen can be put between them, on which an intermediate image is formed, providing insensitivity to projector, but with much lost light. Finally, a large Fresnel lens with a long enough focal length can be used as a field lens, producing a brighter image with a field as large as the Fresnel lens.

\section{Field Lens and the Eye}

In a variation of the above, attempt to view the aerial image formed by a projector. Next add a screen and then a Fresnel field lens. The observer's eye lens and retina then act as the second projector and platen screen in the above demonstrations. For direct viewing, greatly reduce the amount of light, so as not to damage the eye.

\section{MOIRÉ}

Moiré phenomena are particularly easy to demonstrate. There is extensive literature on moiré, and almost anything involving flat transparencies works on the projector, including many everyday objects, such as screens, cloth, perforated sheet, and combs. A kit sold by Edmund (\$6) is useful. For the projector, two should be obtained. Use the variable enlarging feature of the photocopier to make patterns with different periodicities to show phenomena related to beating and group velocity.

Hexagonal patterns, which are not commonly used for moiré, provide excellent effects. These patterns can be produced on the computer. Another source is perforated metal. An interesting effect is obtained with a relative rotation of two such pieces, about $250 \mathrm{~mm}$ on a side. Put a pin in the center of the lower of the two, so the upper one can rotate around it. The smoother sides of the punched sheets should be in contact.

Moire is usually demonstrated black and white, but fascinating color effects can be obtained. For example, overlay two checkerboard or bar transparencies with different pairs of colors. Try a pair of identical hexagons, each consisting of the subtractive primary colors.

Show applications of moiré in metrology and alignment, where small motion produces a large change in appearance. Overlay and translate equally spaced lines, concentric circles, etc. Moiré accentuates the difference between two nearly identical patterns, a technique used in optical inspection.

Separate one sheet from the other, possibly tilted, to show what happens. Show that a coarse moire pattern is insensitive to defocus, although the individually contributing patterns are blurred. With two projectors, overlap on a screen the images of two transparencies. If the images of each are resolved, a 
moire pattern results, but if they are not, no pattern will be seen. Show the moire pattern that arises between the projector Fresnel lens and a transparency above it.

\section{SCATTERING}

\section{Surface Scattering}

A piece of ground glass on the platen produces a dark image because it scatters light beyond the lens. Accordingly, when viewed from near the platen, the ground glass appears lighter than its surround. Put two different types of diffusers side by side on the platen, and compare brightnesses on the screen and brightnesses viewed from near the lens. Show that the scattering results from a combination of a roughened surface and a refractive index mismatch between air and glass. Put the ground glass in a petri dish and pour in water to reduce the scattering by more nearly matching the refractive index of the glass. Use mineral oil for a better index match and still less scattering.

Linear structures scatter most efficiently in directions perpendicularly to themselves. Make an object to demonstrate this by abrading a piece of plastic sheet, about $100 \mathrm{~mm}$ on a side, mostly in one direction. This can be done manually or by holding the plastic against a belt sander in one orientation, producing a number of scratches in roughly the same direction that do not have the regularity of a grating. Block the platen, except for a small centered hole, and hold the plastic piece at the projection lens to produce a scattering distribution perpendicular to the lines. Rotate the scattering piece and the scattered light distribution rotates with it.

\section{Particle Scattering}

Show particle scattering by dropping particles into the beam leaving the projection lens. Partially block the platen to confine the beam, and darken the room by projecting the light against an absorber. Blackboard erasers can be beaten above the beam or baking flour can be sprinkled in. Any kind of safe dust can be used. Cigarette smoke works, as does water droplets from dry ice.

\section{Rayleigh Scattering}

Use a flat-bottomed, clear-sided vessel nearly tall enough to fill the space between the platen and the projection lens. Flat bottomed jars are rare in the supermarket, but chemistry glassware can be used, or a vessel can be made by gluing a flat piece of clear plastic, enlarged to be a stable base, to the end of a clear plastic tube. Cover the platen except for an opening smaller than the vessel, located where the light from the platen is most perpendicular to it. Nearly fill the vessel with water and add a small amount of milk or non-dairy creamer. The amount needed must be found experimentally, and is best added with an eye dropper. The scattered light appears as a column in the liquid. Hold a polarizing sheet beside the vessel and rotate it to show that the light is polarized with the E vector horizontal. Too much milk results in multiple scattering, which gives unpolarized light. Hold a polarizer above the vessel to demonstrate that the unscattered light is not polarized.

The unscattered light, projected to the screen is reddish because of the wavelength dependence of Rayleigh scattering. To emphasize the color change, use an additional hole in the field stop to transmit light that does not traverse the scattering liquid. Since the projector lamp has a lower color temperature than the sun, the scattered light does not look blue. Add a color correction filter to enhance the blue.

To show what happens when the incident light is polarized, place the vessel on a linear polarizing sheet on top of a rotation table with a central hole. Rotate the entire system, and the irradiance, as viewed from a point in the audience varies. Nearby observers might need to close one eye to maximize the contrast.

Liquid scattering experiments should be set up in advance optimize the formulation. It may also be necessary to get rid of air bubbles on the sides of the vessel, which can be removed with a long stirring rod. 


\section{Other Scattering Demonstrations}

Show multiple scattering by adding more milk. As the amount is increased, there is less and less transmitted light, and the column of light first seen in the vessel becomes a general glow. With a certain amount of milk, the numbers of singly and multiply scattered photons are comparable, in which case rotating a polarizer outside the vessel shows that some of the light is polarized and some is not. With still more milk, virtually all photons are multiply scattered and rotating a polarizer shows nothing. Observe forward scattering by standing over the projector and looking down at a scattering beaker.

Scattered light can be observed in order to study dissolution and flow. For example, if an eyedropper of milk is added gently to water through which light from the projector base passes, the slowly changing distribution of the milk can be seen.

Show concealment by scattering, such as occurs with fog, turbid water, and paint. Put a petri dish of water above a transparency on the platen. Add various amounts of milk to the dish and observe the disappearance of the transparency's image. Alternately, arrange several petri dishes with the same amount of water and different amounts of milk, or with the same amount of milk in different amounts of water. Tilt a petri dish to show the variation of concealment with thickness. A transparency with different feature sizes shows that fine features are affected more than the coarse ones.

\section{FLUORESCENCE}

Fluorescing substances are readily available. These include "day-glow" paper, posterboard, markers, and red plastic drafting tools. Some laundry powder fluoresces, as does its water solution. Another liquid is Prestone antifreeze, which should be diluted in water. Plastic sheets are available from distributors, who might have scraps. There is luminous paint, e.g. Edmund F31,806 (\$10), and safety tape sold by industrial suppliers. Chlorophyll can be extracted from plant leaves.

From a magenta-looking fluorescent plastic sheet, cut out a disc or square about $100 \mathrm{~mm}$ across, and smooth the edges. This can be done with woodworking tools. Hold this piece under the projection lens and orange light is emitted. It is most intense at the edges because the fluorescent light is emitted in all directions from points within the plastic, and much of this light is guided by total internal reflection to the edges of the plastic piece. To show that there is a wavelength change, put a green filter over the platen, and orange light still appears. To demonstrate that the fluorescent plastic absorbs, put it on the filtercovered platen to produce a black image on the screen. This last demonstration does not work if the excitation is by the UV. Let the light that has passed through one fluorescing sheet pass through a second one, which emits less. Try fluorescers with different absorption bands in series, and switch their orders.

To show that absorption varies with wavelength, put side by side on the platen strips of red, green, and blue transparencies. Put a piece of fluorescent plastic across these strips, covering half of each. It is seen on the screen that the light passing through the three is diminished differently by absorption. (Note here that what is perceived as one color may have other spectral components. This can be checked with the spectroscope described below.) Fluorescent plastic itself can be used as a filter in this demonstration.

With a sheet polarizer, show that the plastic fluoresces regardless of the polarization of the incident light, that the polarization of the nonabsorbed light is not changed, and that the emitted light is not polarized.

Fluorescent posterboard, paper covered with fluorescent paint, paper marked with fluorescent markers, etc. can be held against the screen and excited by the projector light. Analogues of the above experiments can be done with transmitting color filters on the platen.

\section{GRATING}

The best grating period for overhead projector demonstrations is about $10 \mu \mathrm{m}$, i.e. 100 lines per millimeter. One such grating is the Central Scientific 8648001 (\$30). Most gratings sold for education purposes have too small a period, so the diffracted light falls beyond the screen, and the light is too spread 
out and too dim. Mount the grating in a square piece of cardboard about $100 \mathrm{~mm}$ on a side to obstruct light that does not pass through the grating. Hold the grating near the projection lens, both before and after it. Many gratings have equal lines and spaces, so the there is little light in the even number diffraction orders, except for the zeroth.

\section{Basic Grating Properties}

Cover the platen except for a hole about a centimeter in diameter and hold the grating after the projection lens. Observe the diffraction orders and the sequence of colors. Rotate the grating about the central ray to swing the orders in an arc. Tilt the grating in the principal plane perpendicular to the orders to produce asymmetrically separated orders, so that, for instance, the distance between the 0 and +1 orders differs from that between the 0 and -1 orders. Tilt the grating in the principal plane parallel to the lines to produce orders that intersect the screen on an arc. (In direction cosines, everything is linear.)

Use gratings with different periods to show how the separation of the orders changes.

Hold a grating under the projection lens and move it up and down, changing the separation of the orders on the screen, which can be thought of as images of "virtual orders" that are backward extensions to the platen of the diffracted light.

Replace the field stop with a hole with one with a slit for more light. Compare prisms and gratings, showing the difference in the sequence of colors and that gratings can provide more dispersion.

\section{Multiple Gratings}

Put one grating after another to produced an array of orders of orders, each order of the first grating giving rise to multiple orders of the second. Rotate the gratings relative to one another to skew the pattern. If the grating lines are perpendicular, the array is square for gratings of the same period, or rectangular if the period are different.

A number of interesting effects can be produced with crossed gratings. Mount the crossed grating under the projection lens so that it can be rotated about its axis, and put a turntable with a central hole on the platen. Place various objects on the turntable and rotate both them and the grating. With a cardboard that has a single slit cut out, a pattern like that of a water wheel is produced. Perforated metal gives interesting patterns. Another object is a cardboard with random holes. The image is a messy collection of shapes and colors that can be used as an example of the common scientific endeavor of decomposing a confusing situation into pieces that can be understood. Insert color filters to show that the pattern can be spectrally decomposed. Block out all but one of the holes to show spatial decomposition. Combine the two decompositions. A good perpendicular crossed grating is the "Rainbow Symphony Spectrostar" (\$8).

With a polarizer, show that diffraction angles do not vary with polarization, although the amount of light may vary.

\section{Color Addition}

A grating can also be used as a means to demonstrate some color addition phenomena. With multiple holes in the field stop, different orders can be overlapped in various ways to produce additive color effects. To add spectra that are reversed, cut two parallel slits about $50 \mathrm{~mm}$ apart in a cardboard that is placed on the platen. Above the platen, hold a grating with the lines oriented parallel to the slits. Move the grating up and down to cause the +1 order from one slit to overlap the -1 order from another slit, adding red and violet to give magenta.

\section{SPECTROSCOPE}

Make a spectroscope by mounting a grating after the projection lens and covering the platen except for a slit, whose position is chosen for best viewing on the screen. Place various colored objects on the slit and 
observe their spectra. The zero order shows their appearances, providing qualitative demonstrations in color vision, showing, for example, that something that looks yellow may contain other colors. Show the tradeoff between resolution and power with slits of different widths or with a wedge-shaped slit. The narrower the slit the more saturated the colors, but the less light.

Among useful objects are: Colored plastic sheets and color filters. Magenta is instructive, as is yellow, which may contain red and green. Transparency markers on transparencies work. Didymium glass (available from Rolyn Optics and elsewhere) has distinct absorption bands, and fluorescent plastics usually have a single dark band. Interference filters can be tilted to change the color of the light they transmit. Colored liquids can be put in petri dishes or the like.

With an unsaturated colored plastic sheet, make a staircase of one thickness, two thicknesses, and so on. In the zero order the color becomes more saturated, and the spectrum become more confined.

Do side-by-side comparisons by putting samples next to one another on the platen slit. Overlap samples for color subtraction.

Do "astronomical spectroscopy" by using the projector in reverse. Place a white paper screen on the platen and a grating at the lens. Look out a window at night to observe the spectra of star surrogate sodium and mercury street lights.

\section{INTERFERENCE COLORS}

Interference colors can be shown on the overhead projector. Put a transmitting interference object above the platen and observe the color of its image. Tilt the object to change the color. Some objects are polarization-sensitive enough that the colors can be changed by rotating a polarizer before or after some them. The platen can be blocked except for two or more holes to compare with white light or with light that has passed through another filter.

Reflect light directly from the object to the screen. This light is not imaged, so a blur is produced, but it is colored. If the filter absorbs little, the transmitted and reflected colors are complementary, and the filter can be oriented to overlap the two beams, giving a white sum.

Inexpensive filters are Edmund color separating dichroic filters, $\mathrm{P} 43,45 \mathrm{X}$, where $\mathrm{X}=4,5,6,7,8$, about $\$ 12$ each, and American Science \& Surplus dichroic filters, about $\$ 5$. "Iridescent" gift wrapping sheets show angular dependence. A thin film deposition company may be able to provide rejected material that is excellent for qualitative demonstrations.

Most everyday examples of interference are in reflection, e.g. thin oil films in parking lots. To observe reflection interference colors, block the platen except for an off-center opening and use a mirror to reflect light to a tilted object and thence to the projection lens. This requires a lot of apparatus.

\section{POLARIZATION}

Polarization phenomena are dramatically and colorfully demonstrated. Most demonstrations employ sheet polarizer, which need not have the highest extinction. In general, avoid colored polarization material, so the colors arise entirely from wavelength-dependent phase shifts. Both linear and circular polarizers are useful, and circular material can be reversed and used as linear polarizer. Sheet waveplates are also useful. Some material is available in small, inexpensive pieces. Material that can be had only in larger sheets can be cut up and divided between a number of people.

\section{Basic Polariscope Arrangement}

Convert an overhead projector into a polariscope as follows: Set up a rotatable polarizer on the platen. Above this, put a rotation table to hold the object under study. Topmost is a rotatable analyzer. Separate the turntables with thin wood sheets with holes. Rubber bottle stoppers, which have a lot of friction, are 
good for this. The analyzer can be mounted on such a turntable or on a separate stand that holds it under the lens. All the apparatus should be located between the platen and the projection lens, since the projection lens/mirror assembly affect polarization. Locate color filters above the analyzer or below the polarizer, since they may be birefringent. Make comparisons by putting objects side by side or by leaving an empty space next to the object being observed. To show the orientations of polarizer, analyzer, and the objects between them, make pointers of birefringent plastic that show up, in general differently colored, for all orientations.

Show that the projector lens/mirror assembly affects polarization by placing a polarizer on the platen and an analyzer alternately before and after the lens.

\section{Two Polarizers}

Show the change of transmittance with the relative angles of two polarizers. Show that there are two extremes of light and dark per revolution.

\section{Three Polarizers}

Set the polarizer and analyzer to extinction and introduce a third polarizer between them at an arbitrary angle, resulting in light at the screen. Rotate the inner polarizer, giving four cycles of brightness per rotation. Similarly, set the outer polarizer and analyzer parallel, and insert between them another polarizer that reduces the light, and whose full rotation gives two cycles of irradiance.

\section{Determining Polarization Orientation with a Half Shade Device}

When an analyzer is rotated relative to a polarizer, it is difficult to find the positions where the two are parallel and perpendicular, since the irradiance is an extremum. The positions can be found more easily and more accurately with the half-shade device. Cut a piece of polarizer so one reference edge makes an angle with a principal direction. Cut two pieces from this one. Flip one over and contact the two portions of the original reference, so the principal directions of the two halves angles with the common edge. When this pair is rotated in linearly polarized light, there are four angles at which the two halves are equally bright, when the edge is perpendicular and when it is parallel to the direction of polarization. This is an example of differential detection, in which the eye compares the brightness of adjacent fields, which can be done to do a few percent.

\section{Multiple Polarizers and Analyzers}

A variety of effects can be obtained by juxtaposing pieces of polarizer cut at different angles relative to their principal directions. With orthogonal pieces acting as a dual polarizer, followed by, say, a piece of plastic and an analyzer, two relative orientations can be seen at once. Similarly, strips of polarizer cut at different angles can be put together. With similar analyzer strips at right angles, a matrix of polarizer and analyzer angles is produced.

\section{Waveplates}

Waveplates, or birefringent plastic sheets, can be put between polarizer and analyzer and rotated. Such waveplates have a color dependence, which can be suppressed with a color filter or demonstrated using white light or possibly with different color filters side by side on the platen. Show that there are two principal directions by rotating a waveplate between polarizers. Show that two quarter wave plates in the same orientation acts like a half wave plate, that orthogonal identical wave plates cancel, and so on. An array of waveplates of the same type oriented differently makes an interesting display.

\section{Circular Polarizers}

Make a circular polariscope by replacing the linear polarizer and analyzer with circular polarization material, usually a left-handed and a right-handed pair. Such a pair would ideally extinguish light, regardless of their azimuths, but in practice there is chromatic variation, and demonstrations work best for a particular relative azimuth. Suppress chromatic effects with a color filter transmitting around the design 
wavelength of the circular polarizers. Place various objects in this polariscope and compare their appearances with those in the linear polariscope. Rotate the objects. Show that a linear polarizer and a quarter wave plate make a circular polarizer.

\section{Calcite Crystals}

Calcite crystals are usually not as large as is desirable. One way to help is with an auxiliary lens or with an enlarging unit designed to show $4 " \times 5$ " transparencies on a projector. Make a small hole in a piece of cardboard and place the crystal above it, resulting in two spots. Rotate the crystal and rotate polarizers before and after. Add a second crystal to split each of the spots into two more spots, depending on the orientation. To make a thicker crystal, put one on top of the other, possibly with index matching material. The orientations are matched when the combination produces two spots rather than four. A good buy is Ward's $49 \mathrm{E} 1602$, a $4 \mathrm{~cm} \times 4 \mathrm{~cm}$ crystal for about $\$ 18$.

\section{Mica}

Hold a piece of mica between polarizer and analyzer and change its orientation to show interference colors.

\section{Stress Birefringence}

Many materials are birefringent when stressed. Polycarbonate, sold under various trade names such as Lexan ${ }^{\mathrm{TM}}$, is very sensitive. Pieces of polycarbonate can be twisted and bent by hand between polarizer and analyzer, and models of structures, e.g. bridges or buildings, can be made and stressed in various ways, for example with thumb screws. Many plastic sheet products that are stretched during manufacture have permanent stress, observed qualitatively by rotating them between polarizer and analyzer. Many plastic objects, such as rulers, protractors, CD boxes, and food containers are birefringent. Try all clear plastic objects. With molded plastics, differential cooling results in stresses, so the location of the mold gate can be seen. Plastic bars, plates, and other shapes may have birefringence resulting from their manufacture. For example, some cubes show the same kind of pattern in two orientations, but a different one in the third, suggesting that they were cut from an extruded bar. Some bars show different patterns in all three directions, suggesting that they were cut from plates. Stress lines can be mapped by setting the polariscope with crossed polarizer and analyzer and projecting the pattern on a white screen where the blackest regions are marked. The polarizer and analyzer are then rotated a little together and the dark lines are marked again. Continuing this process maps the stress lines. This procedure and others are discussed in books on photoelasticity.

\section{Optical Activity}

The polarization azimuth of linearly polarized light rotates as it passes through an optically active material. Light Karo $^{\mathrm{TM}}$ corn syrup is a readily available optically active material. Karo syrup is water soluble and easy to dispose of. It keeps for a long time, and can be reused. Pour the syrup in advance, since there may be bubbles that float upward very slowly. Place a vessel of the syrup about six inches deep with a fairly flat bottom between polarizer and analyzer, producing a color from incident white light. Rotating the vessel has no effect. Put a color filter before or after the polariscope and rotate the analyzer to show that light of the wavelength being viewed is nearly linearly polarized, but at a different angle. Switch to a different color, which is also linearly polarized and rotated, but at a different angle, so dispersion causes the color change with white light incident. Two colors can be compared simultaneously with two filters, each blocking half the beam. Compare vessels with different depths of syrup. To show continuous thickness variation, cut a strip of sheet polarizer in a principal direction and put it in the vessel at an angle. Remove the polariscope polarizer, and rotate the analyzer, giving a dark band that moves along the polarizer strip. Orthogonally polarizing strips show bands $180^{\circ}$ out of phase. Between circular polarizers, the syrup has no effect. To demonstrate rotation in both directions, obtain levorotary and dextrorotary sugars from a chemistry supply house. 


\section{Polarization from Reflection and Transmission}

It is possible to show changes of polarization with transmission by holding plastic sheets at large angles between polarizer and analyzer. However, the effect is not dramatic. Multiple sheets can enhance the effect, as in the pile of plates polarizer.

Observe transmission at all angles simultaneously with a plastic dome between polarizers. The dome material must not be birefringent. A large dome is Edmund Scientific C71,710 for $\$ 22$. Another source is the "plastic eggs" that hold small toys in vending machines at supermarkets and so forth. Soap bubbles can be used. With the polarizer and analyzer crossed, observe the dark cross, whose orientation is that of the principal directions of the polarizers. Rotate the dome to show its rotational symmetry. Rotate the polarizer and analyzer together. Set the polarizer and analyzer parallel to give a complementary pattern.

To show changes of polarization upon reflection, use black glass or smooth black plastic to eliminate multiple reflections. Put a polarizer on the platen and a rear projection screen vertically on the front edge of the platen. The plastic is held at an angle of about $45^{\circ}$ to reflect the light on to the screen. The platen must be obstructed except for a small opening, since the light leaves it over a range of angles. Vary the angle of the plastic and that of the polarizer to show Brewster's angle. To compare different polarizations, make a field stop with two or more holes, each covered by linear polarizers at a different angles, including those that give $s$ and $p$ polarizations. Tilt the black material to show that reflectivity varies with incident polarization.

\section{Spectral Analysis Of Polarization Effects}

Analyze polarization phenomena spectroscopically by combining the polariscope and the spectroscope. Cover the polariscope platen with a cardboard with a slit and add a grating after the projection lens.

\section{Conoscoopic Effects}

Sandwich thin birefringent materials between a polarizer and analyzer and hold the stack after the projection lens. This uses the projector as a small source of light with a range of angles. The lamp should be without internal mirrors, and the effect may be enhanced by obstructing the pupil so as to pass only light from the filament. The quality of these demonstrations varies with the projector.

\section{Depolarization}

Observe depolarization by projecting light onto the screen with a polarizer following the projection lens. Observe the screen through an analyzer held at the eye to see that the light is not completely polarized. Try other screen material, including aluminum foil, which is better at preserving polarization. Such screen considerations are necessary for three-dimensional motion pictures based on polarization.

\section{Order of Elements}

The order of elements matters in polarization. Between the polarizer and analyzer place two elements and then reverse their order. In general, the result changes. This is an example of non-commutativity in physics. A related fact is that polarization elements can be mathematically represented by matrices, the product of which represent a series of elements, and matrix multiplication is not commutative.

\section{$180^{\circ}$ Rotation Periodicity}

Between polarizer and analyzer, anything can be rotated $180^{\circ}$ and the appearance is unchanged. Likewise the polarizer or analyzer can be so rotated. Show this with homogeneous material such as birefringent sheet or polarizing sheet. More interestingly, use an irregular looking object, e.g. some stressed plastic. Circle a region of fairly uniform color to keep track of it. As the object is rotated, the colors shift and change, but after half a revolution the color in the marked region returns to what it was originally. 


\section{Principal Directions and Flipping}

Place a flat homogeneous object, for example a birefringent sheet, between polarizer and analyzer and flip it about an axis parallel to the platen. In general the output changes, but there are two special orthogonal directions about which the material can be flipped with no change.

\section{ACKNOWLEDGMENTS}

I have freely harvested published material on demonstrations, modifying them as necessary for the overhead projector. A number of people have made useful suggestions, including Tom Ainsworth, Sam Batchelder, Russell Chipman, Paul Coteus, Paul Doherty, Keith Jenkins, Glen Johnson, John Geievenkamp, John Hayes, Arwin Marathay, Betty Moore, Bill Plummer, Doug Smith, Eberhard Spiller, Richard Sonnnenfeld, Phil Stahl, and Jack Taylor. Additional suggestions are solicited.

\section{SOME SUPPLIERS}

Information as of February, 1993

Ain Plastics 800.431-2451T

American Science \& Surplus 708.475-8440 Evanston, Ill 60202 Inexpensive.

Bruce Pine Mica 704.765-4241

Central Scientific Company (CENCO) 800-26-CENCO Franklin Park, Ill

Edmund Scientific $609.547-3488$

General Science Materials Company 714.534-0985 10123 Crosby Ave Garden Grove, CA 92643

Great American Market 213.461-0200 theatrical supplies

Hudson Glass 800.431-2964 Peekskill, NY 10566 ripply glass

International Polarizer 508.481-7495 Marlborough, MA

Learning Technologies Inc. 800.537-8703 59 Walden St. Cambridge, MA 0214 grating material

Polaroid polarizer division $800.225-2770$

Rainbow Symphony 818.708-8400 6860 Canby Ave Unit 120 Reseda, CA 91335

Rolyn Optics 818.914-5707 Covina, CA 91722

Rosco 914.937-1300, 213.462-2233 Port Chester, NY theatrical supplies

Science Kit \& Boreal Laboratories 800.828-7777 Tonawanda, NY 14150

Solargraphics 501.525-1776 Box 7091J Berkeley, CA 94707 (sold through Edmund)

Ward's 800.962-2660 Rochester, NY

PURCHASED EQUIPMENT - partial list

\section{OVERHEAD PROJECTOR}

Plane mirror, Plexiglas - CENCO $6 \mathrm{~cm} \times 9 \mathrm{~cm} \# 70703 \$ 2$

Fresnel lens - American Science and Surplus, Ward's \$2

Overhead projector accessories - audio visual distributors

Turntable - Rubbermaid No. 2936 at supermarket

MONOCHROMATIC GEOMETRIC OPTICS

Plastic cubes, sheets, etc. - Ain and other distributors

DISPERSION

Prisms of same angle and different material -

Spindler \& Hoyer \#33300 $30^{\circ}$ prism BK7 \$125, \#333310 $30^{\circ}$ prism FK2 \$125

IMAGING

Grid transparency - package of 10 Ward's 15E2911 \$8

Ripply glass - stained glass window supply places, glass shops, e.g. Hudson 
Cylindrical lens - Rolyn Optics \#14.0450\$55 $60 \mathrm{~mm} \times 50 \mathrm{~mm} \times 300 \mathrm{~mm} \mathrm{fl}$

Eyeglasses?

Enlarger - Polaroid \#691 \$200, Buhl \#26-067 \$150

MOIRÉ

Moiré patterns for the study of interference - General Science Materials H-1003 \$12

Edmund Scientific kit

Perforated metal - Small Parts Inc. Miami 305.751-0856

Ronchi rulings - Edmund, Rolyn

SCATTERING

Petri dishes - Edmund

reusable polymethylpentene $\mathrm{P} 35,296 \$ 39$ for 10 , disposable polystyrene $\mathrm{P} 38,026 \$ 17$ for 100

Ground glass - Edmund, CENCO $7.5 \mathrm{~cm} \times 10 \mathrm{~cm} \# 86002 \$ 3$

FLUORESCENCE

fluorescent plastic sheets - plastic distributors, e.g. Ain

One manufacturer is Rohm \& Haas 215.592-3000

INTERFERENCE

Interference filter American Science \& Surplus, Edmund

GRATINGS

For grating demo and spectroscope

Basic grating: $100 \mathrm{l} / \mathrm{mm}$

Also $2001 / \mathrm{mm}, 300 \mathrm{l} / \mathrm{mm}, 600 \mathrm{l} / \mathrm{mm}$

CENCO clear area $45 \mathrm{~mm} \times 45 \mathrm{~mm} \$ 27$ ea. , set for $\$ 91$ \#86247-01

Set of diffraction gratings $100 / \mathrm{mm}, 300 / \mathrm{mm}, 600 / \mathrm{mm}$ and crossed gratings $300 / \mathrm{mm}$ - General Science

Crossed Grating

Rainbow Symphony Astrastar \$8

Solargraphics Jupiter Window (sold through Edmund)

SPECTROSCOPY

Color filters - Rosco, Great American Market, Edmund, etc.

Didymium glass - Rolyn

Fluorescent plastic

POLARIZATION

Linear polarizer sheet - International Polarizer, Polaroid, Edmund, etc.

Circular polarizer - Polaroid

Left hand $605230 \$ 12019 " \times 50 "$, right hand $605229 \$ 20019 " \times 50 "$

Wave plate

Polaroid - big sheets

International Polarizer 4" square, package for 5 for $\$ 25$

quarter wave for $550 \mathrm{~nm}, 400 \mathrm{~nm}, 480 \mathrm{~nm}, 640 \mathrm{~nm}$ and half wave for $520 \mathrm{~nm}, 580 \mathrm{~nm}, \ldots$

Mica - Bruce Pine Mica 704.765-4241

Calcite (also listed as Iceland spar) - Ward's 49E1602 $4 \mathrm{~cm} \times 4 \mathrm{~cm} \times 1 \mathrm{~cm}, \$ 17$

Polarization demonstrator set with calcite crystals, benzoic acid, polarizer - CENCO, Ward's, others $\$ 35$

Karo Light Corn Syrup - supermarket

\section{SOME BOOKS ON DEMONSTRATIONS AND EXPERIMENTS}

M.Cagnet, N.Francon, J.C.Thrierr, Atlas of Optical Phenomena Vol.1 (Springer-Verlag, Berlin, 1962)

M.Cagnet, N.Francon, S.Mallick, Atlas of Optical Phenomena Vol.2 (Springer-Verlag, Berlin, 1971)

R.W.Wood, Physical Optics (Macmillan, New York, 1911; Optical Society of America, 1988)

C.F.Bohren, Clouds in a Glass of Beer. Simple Experiments in Atmospheric Physics (Wiley, New York, 1987) $195 \mathrm{pp}$

C.F.Bohren, What Light Through Yonder Window Breaks? (Wiley, New York, 1991) 190pp

G.F.C.Searle, Experimental Optics (Cambridge U.P., 1925) 357pp

M.Francon, M.Krauzman, J.P.Mathieu, M.May, Experiments in Physical Optics (Gordon \& Breach, New York, 1970) 271pp 
E.Deeson, ed, Light an ASE Lab Book (Asociation for Science Education, John Murray, London, 1975) $86 \mathrm{pp}$

H.Palmer, Optics Experiments and Demonstrations (Johns Hopkins, Baltimore, 1962) 320pp

Newport Research and D.O'Shea, Projects in Optics. Applications Workbook

R.Bruman, Exploritorium Cookbook I (The Exploratorium, San Francisco, 1984)

R.Hipschman, Exploritorium Cookbook II (The Exploratorium, San Francisco, 1980)

R.Hipschman, Exploritorium Cookbook III (The Exploratorium, San Francisco, 1987)

H.F.Meiners, edt Physics Demonstration Experiments (American Association of Physics Teachers, 1970)

R.Ehrlich, Turning the World Inside Out (Princeton University Press, Princeton, NJ, 1990) 216pp

H.Daniel, Demonstratiusversude aus der Optik (Musterschmidt-Verlag Gottingen, 1960)

M.Francon, M.Krauzman, J.P.Mathieu, M.May, Experiments in Physical Optics (Gordon \& Breach, New York, 1970) 271pp

M.Francon, Experiments d'Optique Physique (Gordon \& Beach, New York, 1969) 249pp

R.W.Pohl, Optik und Atomphysik (Springer-Verlag, Berlin, 1967) 347pp

Popular Optics (Edmund Scientific, No 9445, 1974)

Unesco, 700 Science Experiments for Everyone (Doubleday, New York, 1958)

E.Edser, Light for Students (Macmillan, London, 1902)

W.A.Hilton, Experiments in Optical Physics (Hilton, Liberty, MO, 1974)

Demonstration Experiments of Basic Phenomena in Optics (American Optical, Southbridge, MA, 1968)

A.F.Wagner, Experimental Optics (Wiley, New York 1929) 203pp

H.A.Robinson, ed, Lecture Demonstrations in Physics Translated from Russian by K.V.Robinson,

(American Institute of Physics, 1963; University Microfilms, 1976, number OP-23512 \$140) 473pp

J.A.Davis, "Resource Letter PhD-1: Physics demonstrations" American Journal of Physics, v 47, p 835 (1979)

\section{Laser specific}

T.Kallard, Exploring Laser Light (Optosonic Press, 1977) Reprinted by the AAPT

H.H.Gottlieb Experiments Using a Helium-Neon Laser (Metrologic, Belmawr, NJ, 1974)

Experiments in Physical Optics Using Continuous Laser Light Educational Series No.1. Optics

Technology, Inc.

R.S.Sirohi, A Course of Experiments with He-Ne Laser (Wiley, New York, 1985) 103pp

F.McAleese, The Laser Experiments Handbook (McGraw-Hill, New York, 1979)

G.Wright, G.E.Foxcroft, Elementary Experiments with Lasers (Wykham, 1973)

G.L.Rogers, Hand Book of Gas Laser Experiments (Illifee Books Ltd, London, 1970)

R.Pantell, Experiments in Optics with a Laser Source (Optics Technology Inc, 1969)

\section{Kid books}

B.Taylor, Bouncing and Bending Light (Franklin Watts, New York, 1990) 32pp

B.Taylor, Color and Light - Fun with Simple Science (Warwick, New York, 1991) 40pp

B.Murphy, Experiemnts with Light (Lerner, Minneapolis, 1991) 32pp

N.Ardley, The Science Book of Light (Gulliver, San Diego, 1991) 29pp

R.Gardner, Investigate and Discover Light A Franklin Institute Science Museum Book

(Julian Messner, Englewood Cliffs, NJ, 1991) 137pp

The Magic Mirror, An Antique Optical Toy (Dover, New York, 1979)

C.B.Grafton, Optical Designs in Motion with Moire Overlays (Dover, New York, 1976)

F.J.Moore, The Magic Moving Alphabet Book (Dover, New York, 1978)

F.J.Moore, The Incredible Moving Picture Book (Dover, New York, 1987) 